\title{
PROYECTO DE PASARELAS PEATONALES PARA “RENFE-ENSIDESA”. MADRID 1987, ESPAÑA
}

\author{
(PEDESTRIAN FOOTBRIDGES PROJECT FOR “RENFE-ENSIDESA”. MADRID 1987, SPAIN)
}

S. Pérez Arroyo, Arquitecto

Fecha de recepción: $13-X-88$ $565-43$

\begin{abstract}
RESUMEN
Presentamos un proyecto de pasarelas tipo, para resolver el paso de peatones sobre vías de ferrocarril.

El modelo, propuesto para ser adaptado a diferentes condiciones geométricas y de lugar, se ha concebido integramente en acero, material cuyas prestaciones son especialmente perceptibles en luces y sobrecargas como las que se nos plantean.
\end{abstract}

Nuestra intención ha sido conseguir un elemento estructural de la máxima esbeltez, simplificando su aspecto para acentuar su carácter funcional.

\section{SUMMARY}

We are presenting a project for a type of footbridges for pedestrian overpasses of railway tracks.

The proposed model, which may be adapted to different geometrical conditions and sites, is completely made of steel, because this material is adequate to the spans and loads of this type of structures.

We have designed a slender structure of simple appearence in order to stress its functional character.

\section{MEMORIA DESCRIPTIVA DE LAS CONDICIONES DE DISEÑO CONSIDERADAS EN LA PASARELA TIPO, RENFE-ENSIDESA}

La necesidad de construir una gran cantidad de pasarelas de peatones -y en algunos casos permitir el paso de ganado sobre las vías de ferrocarril- ha inducido, a ENSIDESA y a RENFE, el estudio de un tipo con posibilidad de adaptarse a las distintas condiciones que este problema plantea.

El modelo de pasarela peatonal concebido es el resultado de un largo y profundo estudio de las existentes en otros países y de las ya construidas en el nuestro. Se ha tratado de conseguir la mejor adaptada, desde puntos de vista estéticos y funcionales, a las necesidades requeridas. Los dos objetivos nos han preocupado por igual: obtener una imagen simple y homogénea que se identifique con el ferrocarril, y utilizar un material de elevadas prestaciones, como el acero, para proporcionar unos inmejorables acabados y un aspecto de objeto industrial digno de un país avanzado.
Estas razones nos han llevado a proponer la utilización del acero con tratamientos superficiales de protección y acabados de pavimento de goma, que dan una sensación de confort al peatón además de una superficie antideslizante de gran calidad.

Las luces exigidas y las sobrecargas previstas permiten ofrecer una sensación de esbeltez máxima que debe ser, a nuestro entender, la ventaja de trabajar con un material de tan buenas prestaciones como el acero.

La tipología estudiada se adapta, con la misma idea, a los distintos anchos de vías y espacios que es preciso salvar.

Se han resuelto tres tipos fundamentales de pasarelas aptas para salvar los casos más comunes de dos, cuatro y seis vías de circulación ferroviaria, desarrollándose posteriormente y a modo de combinatoria tipológica, diversos modelos. Estos no hacen más que iniciar la gama de posibilidades que puede plantear el problema a resolver y que pensamos debe, en cualquier 

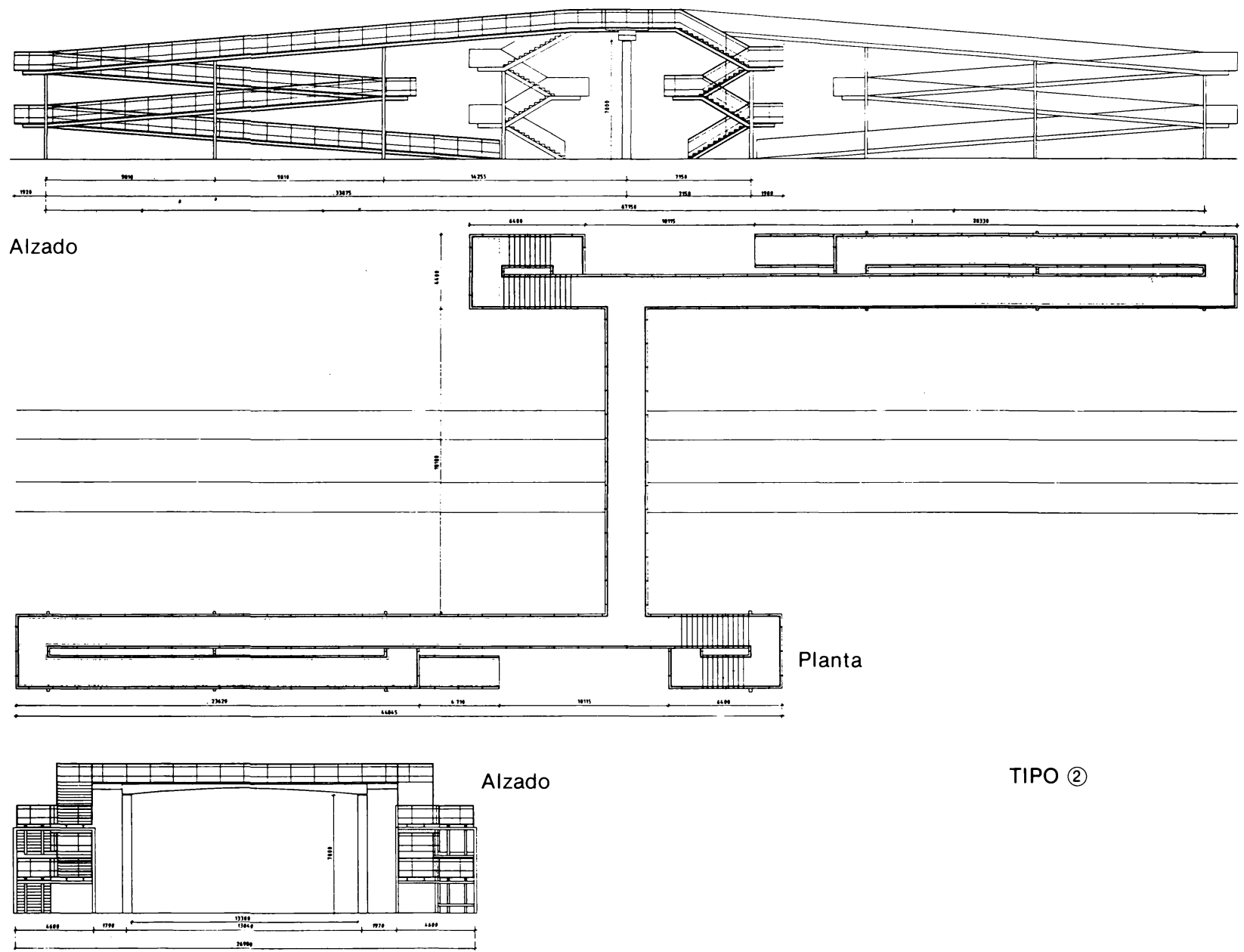

TIPO (2)

Paso a nivel sobre dos vias.

caso, acometerse con un "modelo tipo" único que ofrezca una imagen de orden e información correcta, completa y uniforme a todos los posibles usuarios de estos elementos, en todo el territorio nacional, y teniendo en cuenta la importante repercusión que estas construcciones tienen sobre el paisaje rural y urbano donde se implantan.

Retomamos asi determinadas ideas estéticas de los años sesenta, cuando los proyectos de ingeniería buscaban un resultado acorde con los materiales y los criterios estéticos del momento para todos los objetos de uso cotidiano.

En aquella época, en arquitectura, permanecian de igual modo criterios similares en algunas de las mejores manifestaciones. La escuela de Ulm y el movimiento moderno se mantenían por los arquitectos que pretendieron, como Bakema o Jacobsen, una integración del funcionalismo prebélico dentro de las posibilidades que los materiales modernos empezaban a suministrar.

Hoy asistimos a determinadas manifestaciones, en el diseño de lo construido, revestidas de una gran com- plejidad formal, tenidas de tecnología excesiva en algunos casos, o de vago historicismo en otros. Las razones que nos han llevado a movernos en la línea descrita, se deben a nuestra creencia en la necesidad de retomar parte de aquella actitud más renovadora, haciendo posible una integración de todas las manifestaciones constructivas en una dirección más moderna.

El problema planteado también exigía pensar en la adecuación de las soluciones propuestas a determinados contextos urbanos, que podian estar constituidos por áreas de carácter histórico.

La solución adoptada ha sido también pensada por su contención formal, sin ningún exceso tecnológico, como adecuada a estos problemas de integración. La misma referencia estética de los años sesenta hace que no se produzca con facilidad ninguna imagen de rechazo dada la frecuencia con la que este tipo de soluciones viene asociada a todo tipo de contextos.

La utilización de los espacios de recorrido de la pasarela como espacios urbanos dignos de disfrute y aptos para observar desde éllos el mundo del ferrocarril, nos ha hecho pensar en la incorporación de dos bal- 


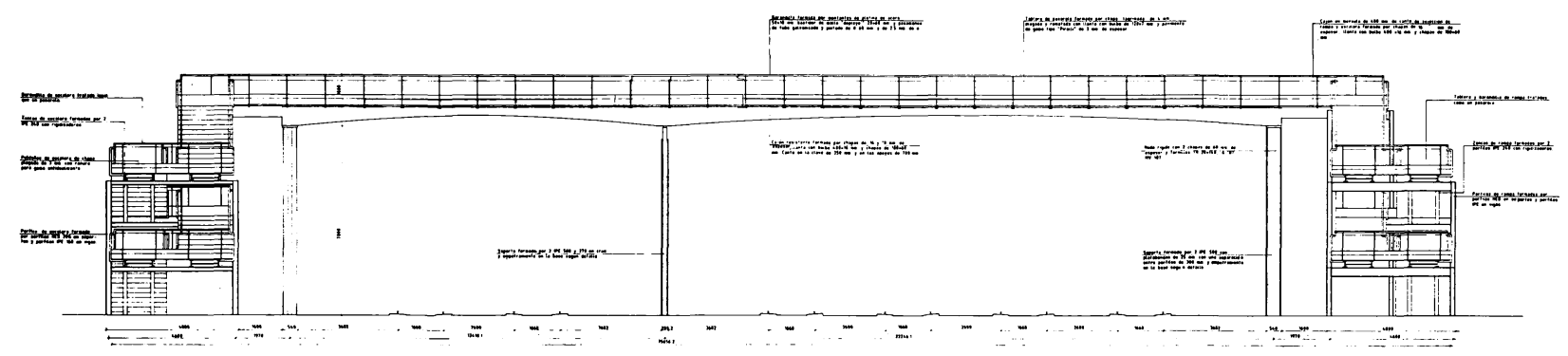

Alzado

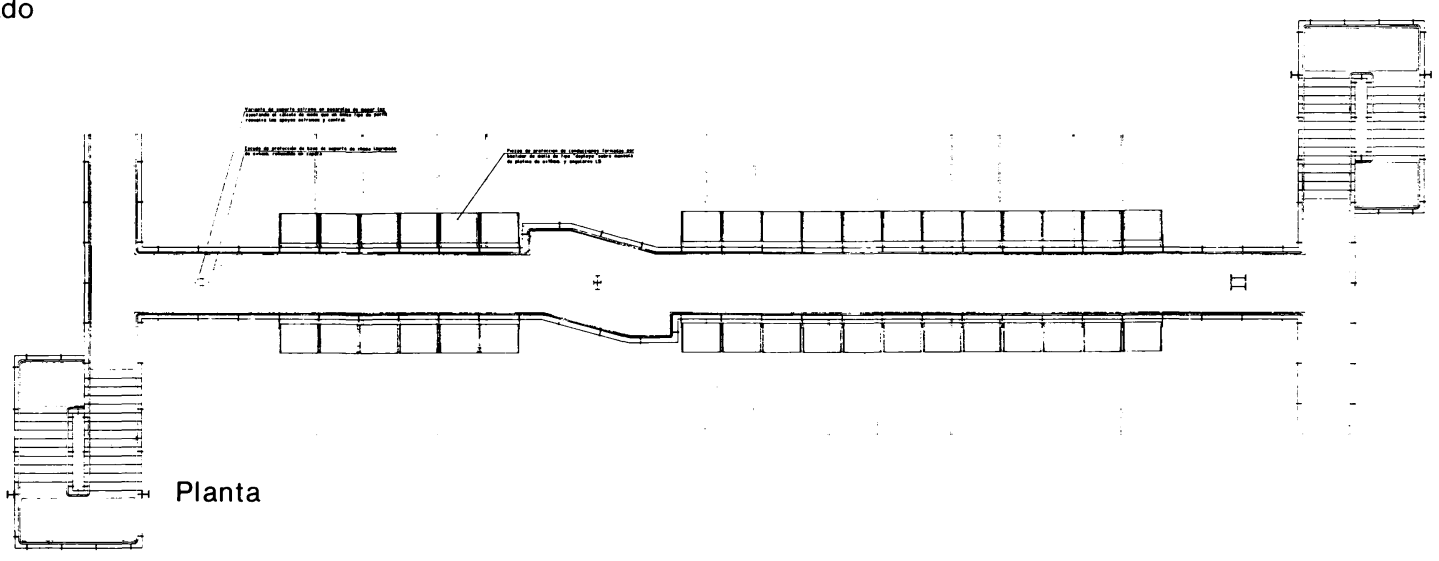

Tipo (2) + (4) con variante de mirador y piezas de protección de conducciones.

cones sobre las vías y en zonas de reposo en rampas y escaleras dispuestas de modo que cumplan esa función de observatorio.

La solución propuesta consiste en una viga cajón constituida por chapas y llantas de bulbo de $400 \times 16 \mathrm{~mm}$, sobre la que apoya el tablero, constituido en este caso por una chapa lagrimada, con resaltes ovalados, de $4 \mathrm{~mm}$ de espesor.

Esta chapa conforma un tablero en sección en "U" sobre el que se coloca en el caso de tráfico exclusivo de peatones un pavimento antideslizante de $3 \mathrm{~mm}$, acoplado en la diferencia de nivel que se consigue con la chapa lagrimada, de modo que los bordes de la goma se protegen de roces y golpes que pueden iniciar el despegue del material. Este tipo de pavimento se puede cortar en la longitud total de la pasarela evitándose juntas transversales.

Las barandillas de protección están previstas con un pasamanos de tubo de $60 \times 2,5 \mathrm{~mm}$ de diámetro, sobre barrotes de pletina de $50 \times 10 \mathrm{~mm}$. Sobre esta estructura se acopla una malla metálica tipo "deployé", modelo 2061, con rombo de $20 \times 60 \mathrm{~mm}$ y $2,5 \times 1,5$ de ancho y espesor. La malla se pliega dando más resistencia, aun cuando la sección de material y el modelo de malla elegido es ya de por sí muy resistente.

De un modo más preciso se aprecian en la sección otros detalles complementarios, como son el remate de la chapa lagrimada conseguido con una llanta de bulbo de $120 \times 7 \mathrm{~mm}$ y el sistema previsto de anclaje de los barrotes de la barandilla al cajón sirviéndose de medio perfil IPE 200. También es preciso recordar que el pasamanos no se encuentra a plomo con los barrotes, sino un poco remetido como sistema de seguridad frente a caídas y por dar una mejor sensación de apoyo que permita observar el paso del ferrocarril.

Se ha estudiado la evacuación del agua de lluvia y limpieza por medio de la pendiente dada, en sentido transversal al tablero, que la conduce a unas perforaciones realizadas en la chapa lagrimada cortando a modo de bocas en el solape de las chapas, donde se consigue el resalte para la goma.

De este modo se evitan igualmente defectos de visibilidad o cualquier tipo de vandalismo.

Para las luces menores y con condiciones teóricas similares de cimentación y topografía, se puede estimar que los costes tendrán una diferencia de un $15 \%$ en el caso de $22,33 \mathrm{~m}$ y de un $18 \%$ en el de $13,8 \mathrm{~m}$.

Las pasarelas están previstas para ser montadas con rapidez y sin interrumpir el tráfico de las vias.

EL hecho de estar concebidas en acero permite que la obra sea en su totalidad prefabricada, de modo que las labores de fabricación y por tanto de soldadura sean, en casi todos los tipos, desarrolladas en taller, con las ventajas de control y ejecución que se derivan de esta característica. 
晋 嘿

Tipo (6) + (A) + (6)

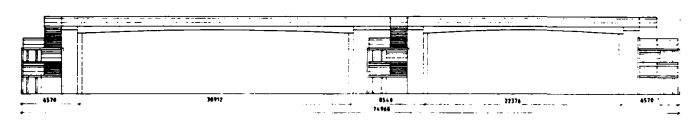

Tipo (6) + (A) + (4)

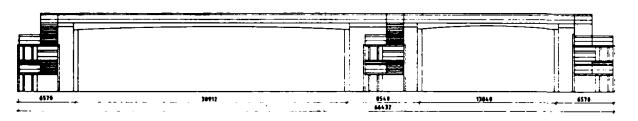

Tipo (6) + (A) + (2)

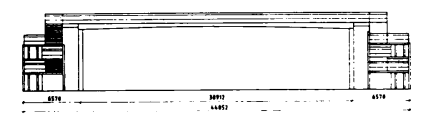

Tipo (6)

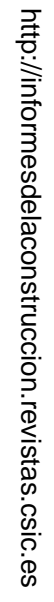

Tipo (4)

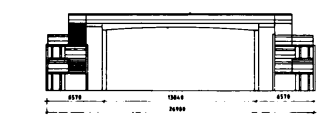

Tipo (2)

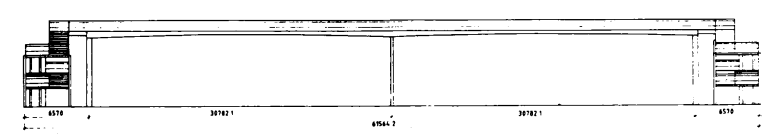

Tipo (6) + (6)

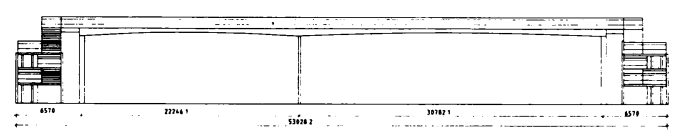

Tipo (4) + (6)

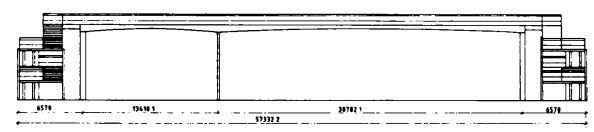

Tipo (2) + (6)
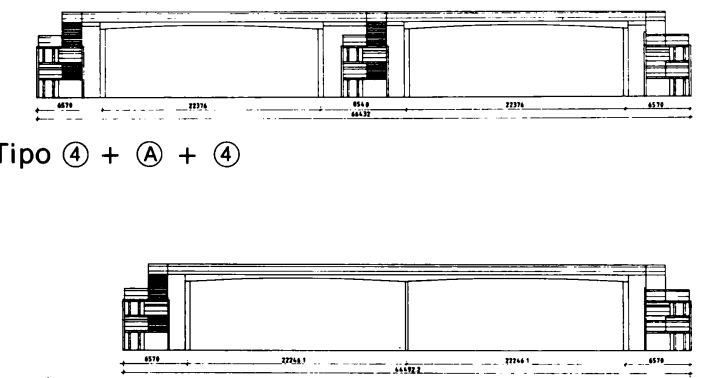

Tipo (4) + (4)

Tipo (2) + (4)

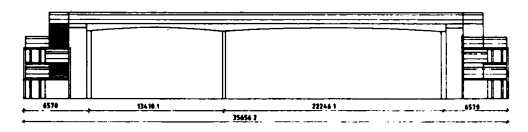

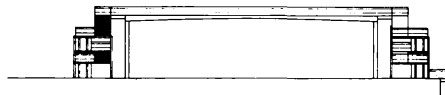

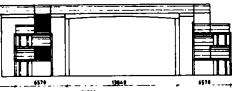

Tipo (6) + (D) + (2)

Tipo (D) + (6)
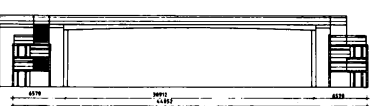

Tipo (1) + (4)

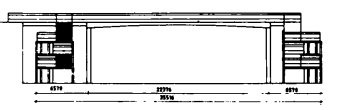

Tipo (1) + (2)

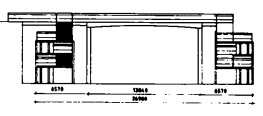

Tipo (1) + (2) 

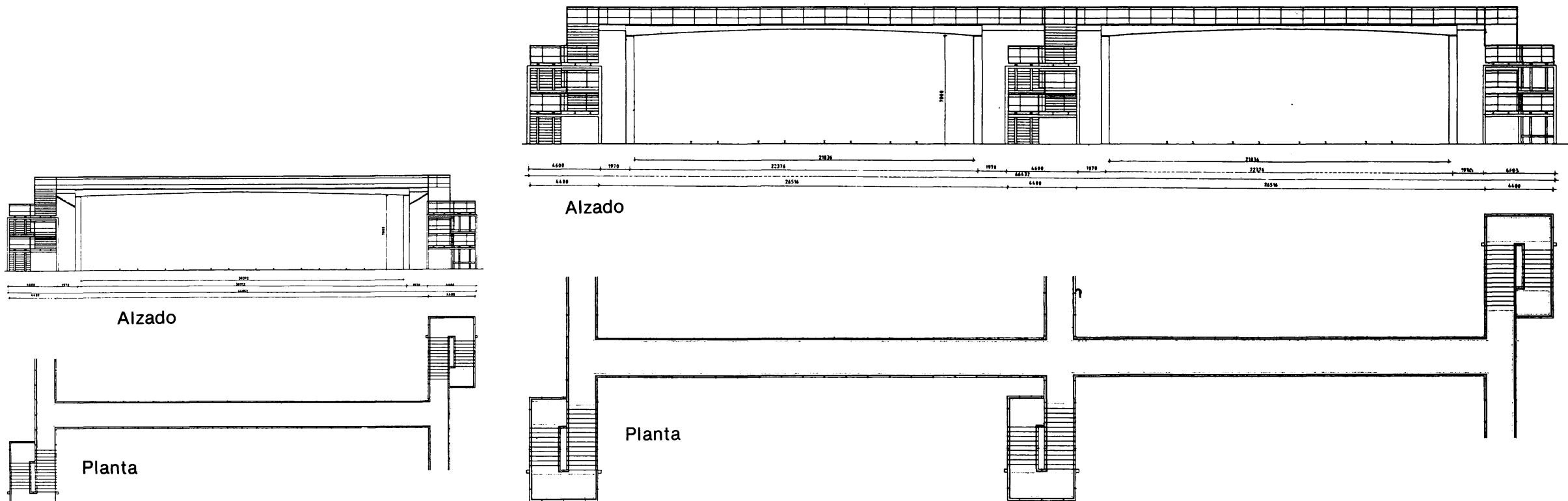

\section{Alzado}

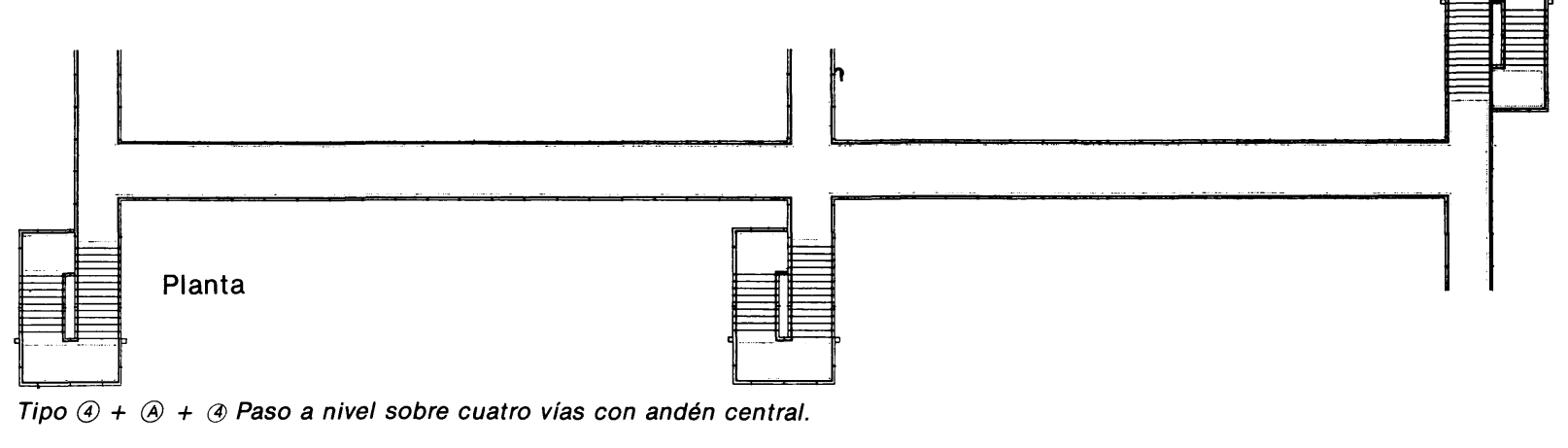

Tipo (6) Paso a nivel sobre 6 vías.

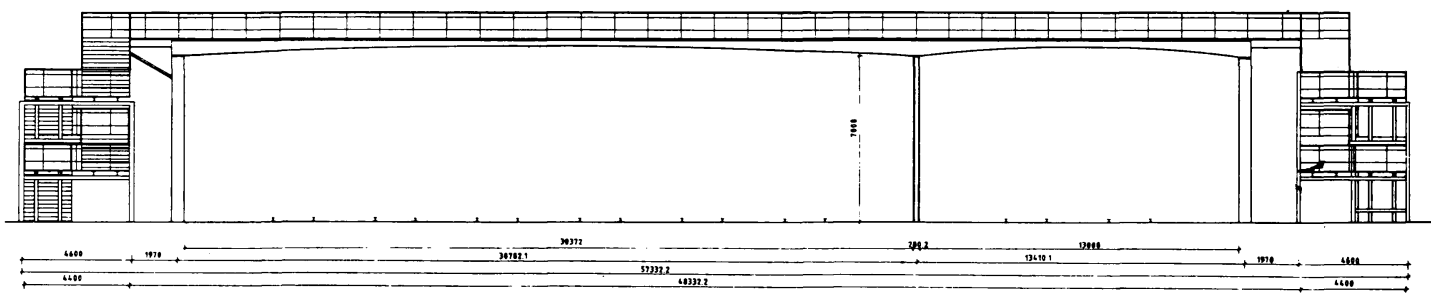

Alzado

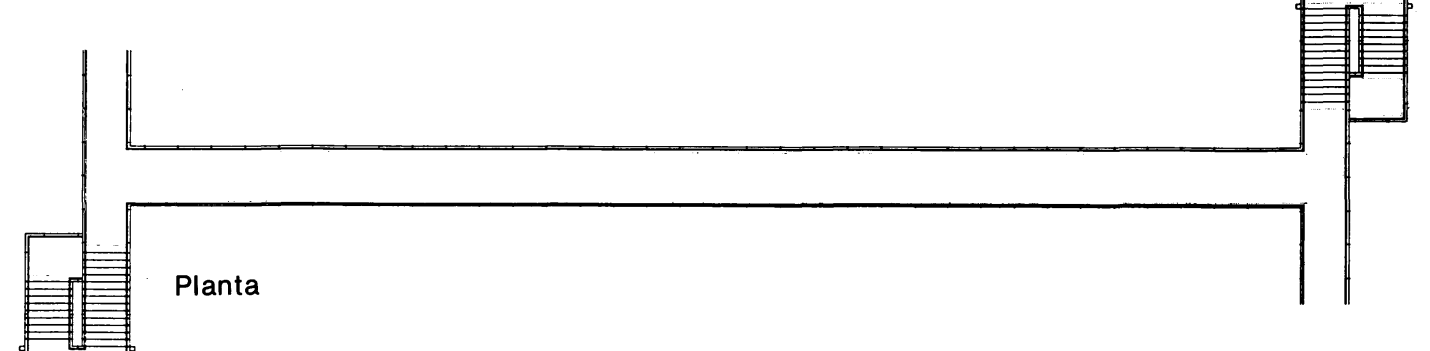

Tipo (6) + (2) Paso a nivel sobre $6+2$ vías con soporte central 


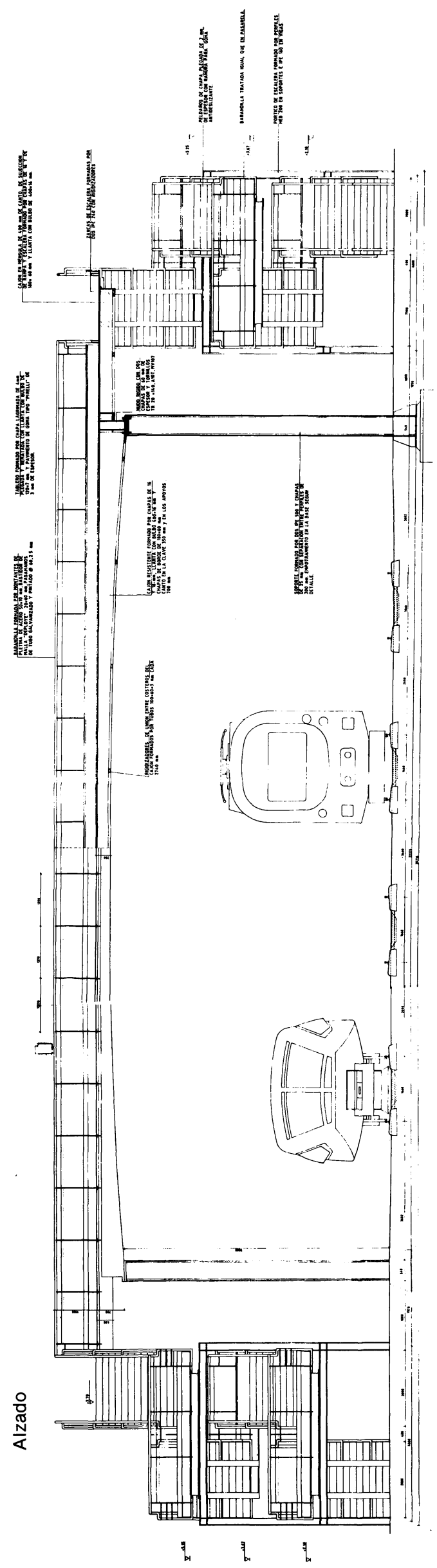

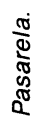

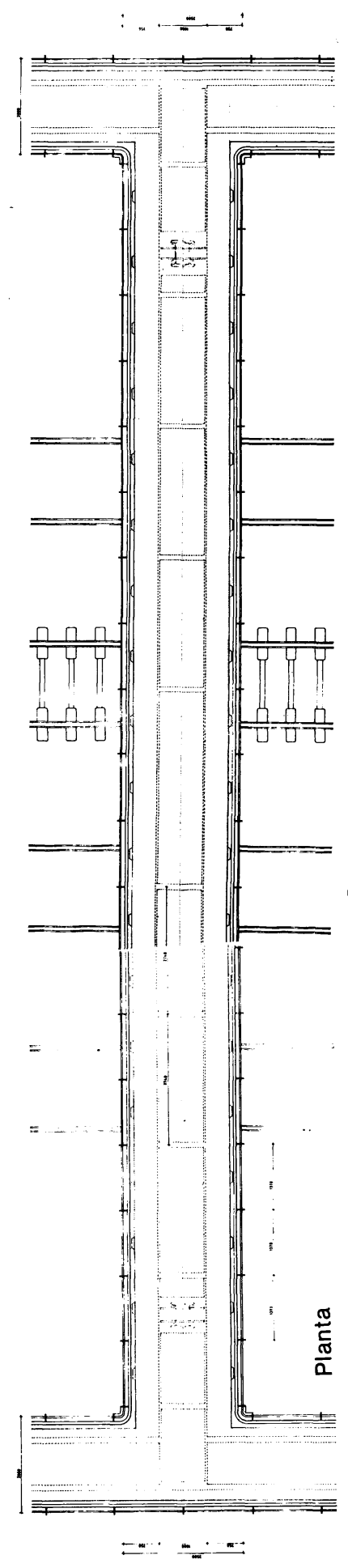




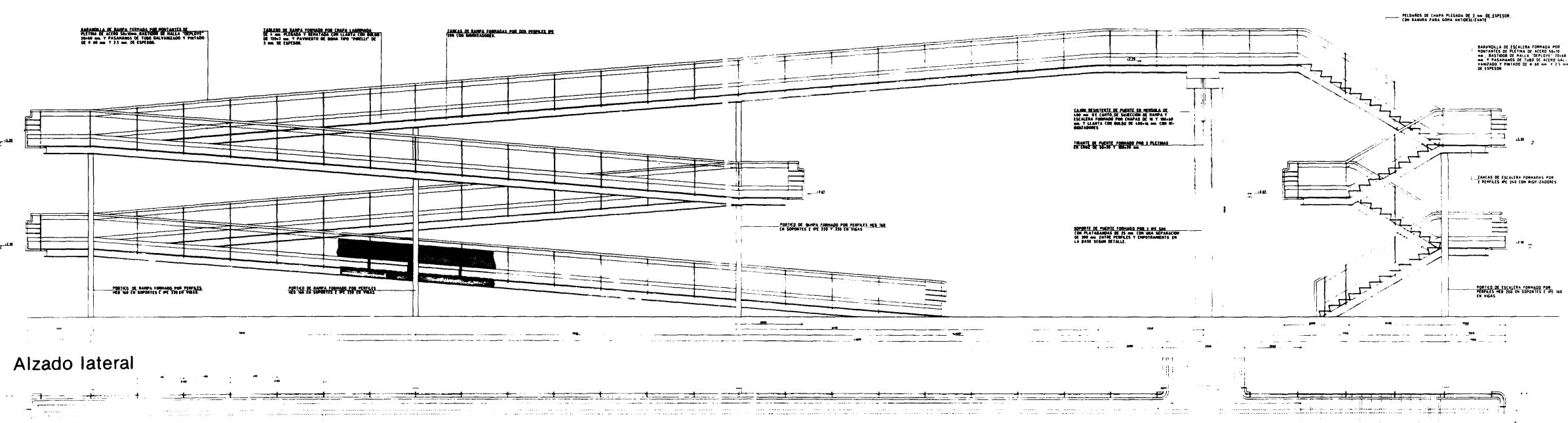
:

$\therefore \quad+$

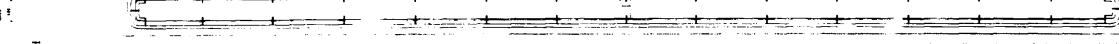

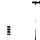

$T_{3}=0+\cdots+\infty=$

Panta
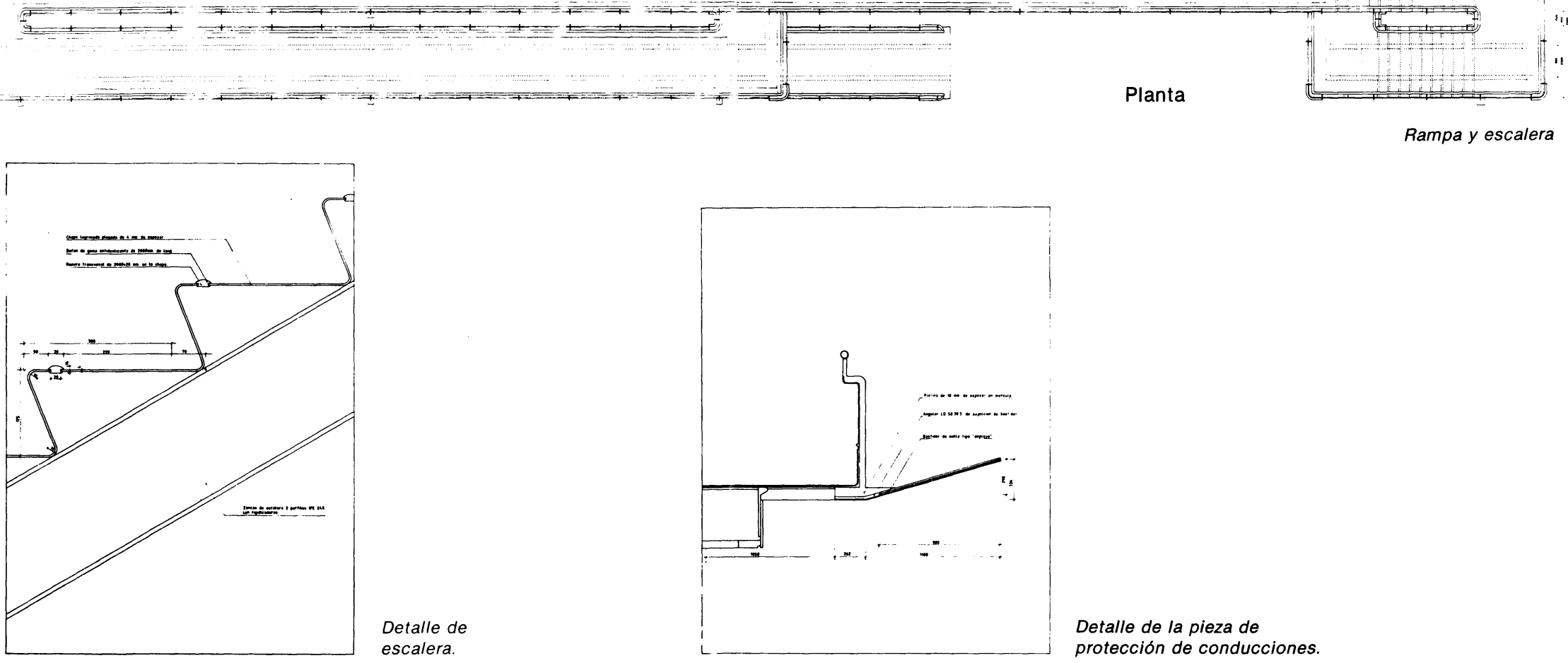

Rampa y escalera 


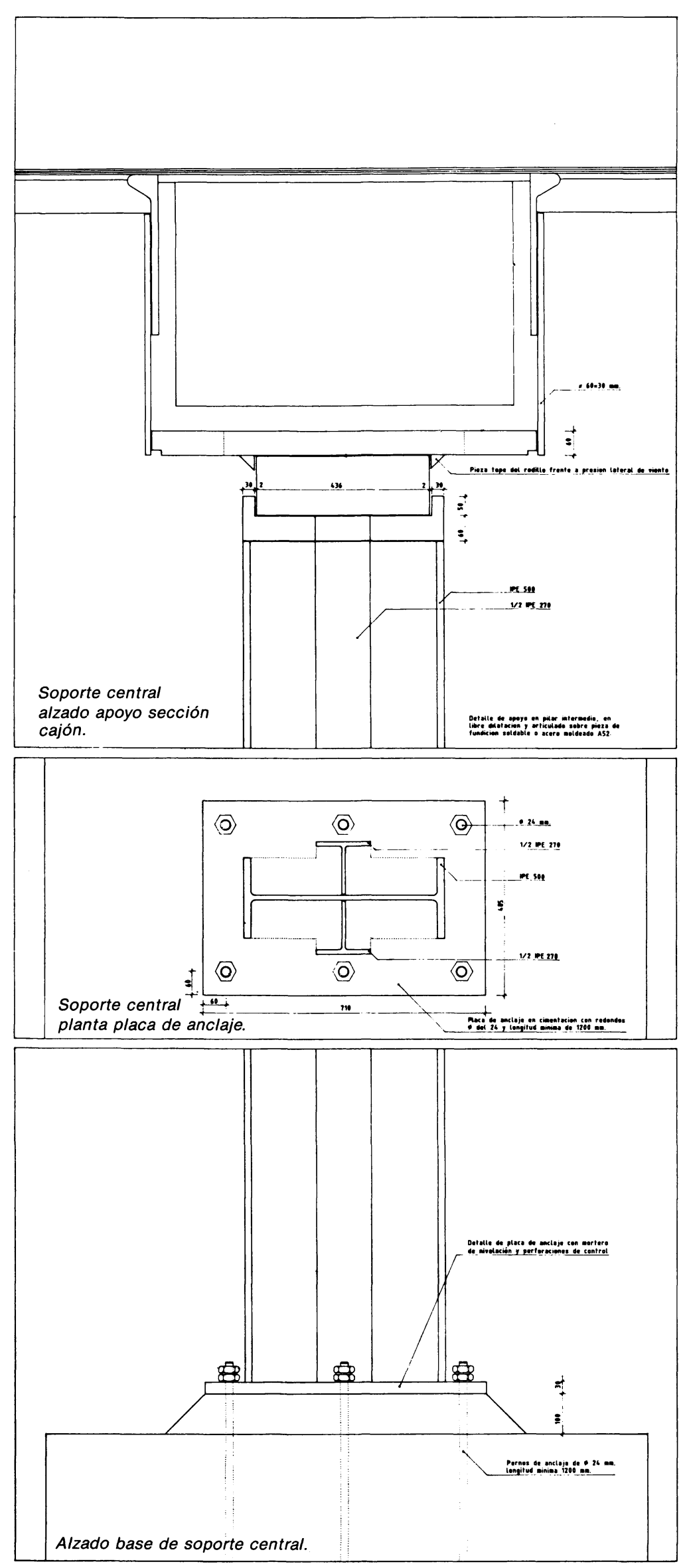

(c) Consejo Superior de Investigaciones Científicas Licencia Creative Commons 3.0 España (by-nc)

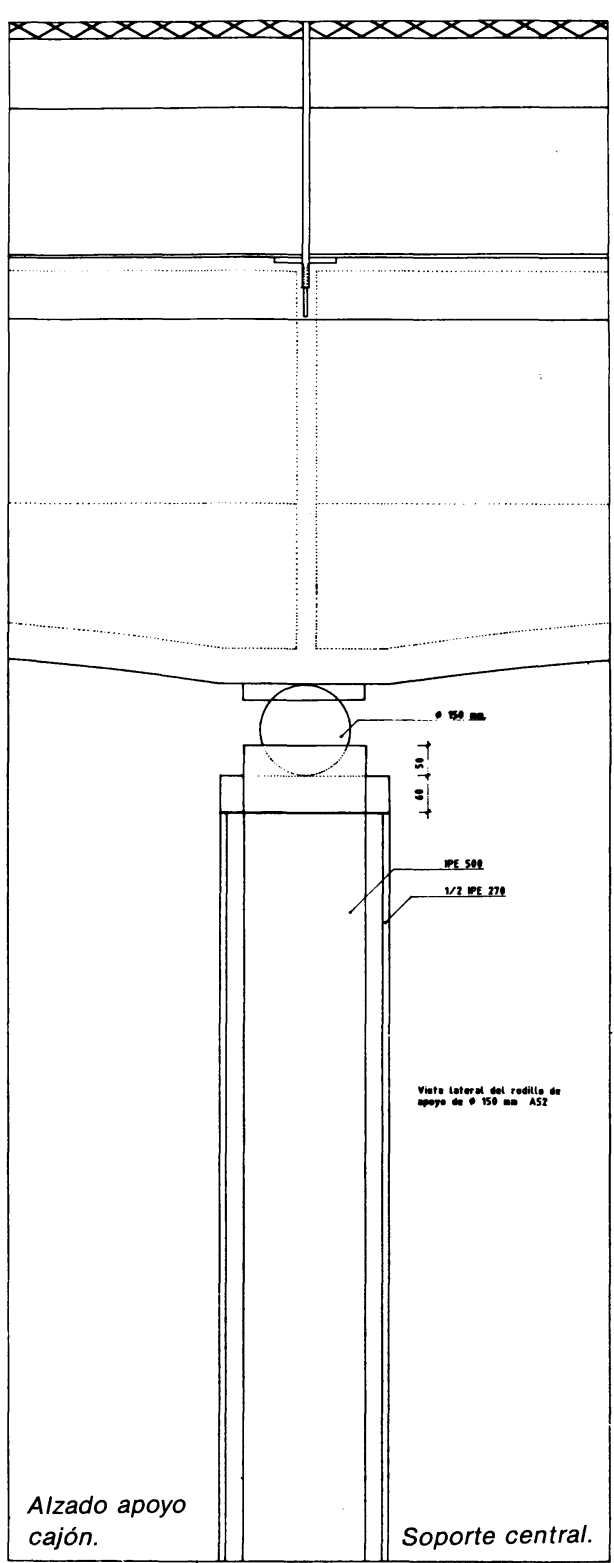

cajón.

Soporte central.

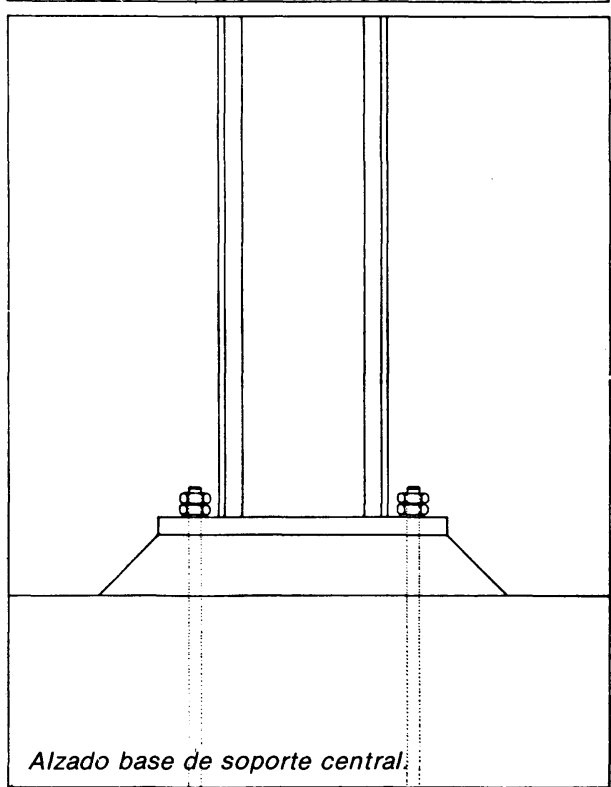

http://informesdelaconstruccion.revistas.csic.es 

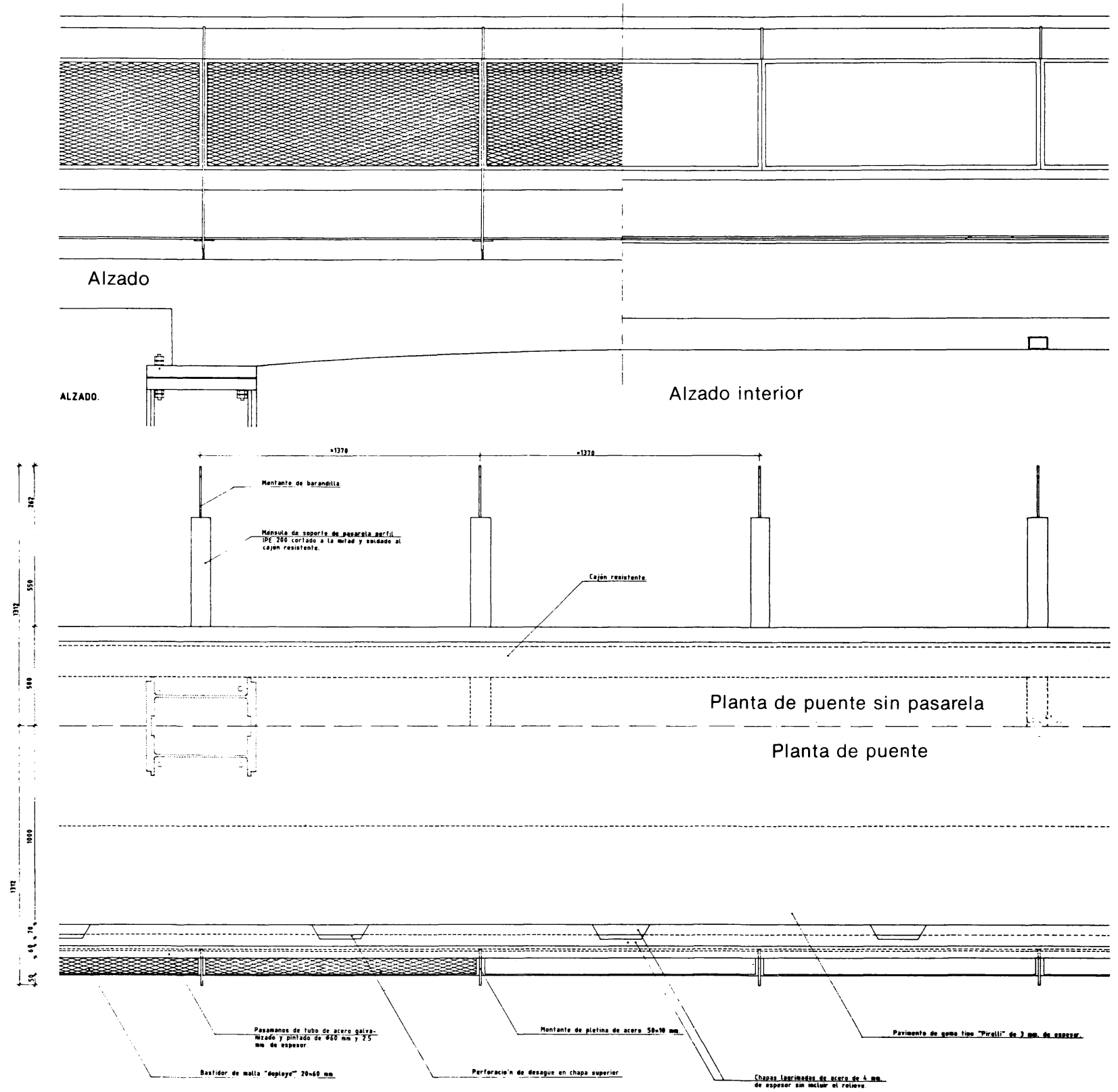

Detalles de la pasarela.

Los enlaces con los pilares y el cajón son rígidos, estando prevista, en el caso de combinarse tramos distintos, la posibilidad de introducir pilares intermedios con apoyos de la pasarela articulados con mecanismos de fundición soldable. Sobre éllos deslizan dos rodillos de acero de $15 \mathrm{~cm}$ de diámetro.

Los pilares de los distintos tipos de puentes pueden ser, como los representados en los planos, concebidos compuestos con perfiles formando un cajón de gran inercia reforzado con chapas o en forma de cruz de menor inercia, igual que la solución presentada en el puente de dos vanos en el apoyo central. El giro de estos perfiles permite ofrecer resistencia a las acciones horizontales en el centro y absober momentos en los apoyos extremos.
Pero debe en cualquier caso estar concebida para resistir las solicitaciones previstas. La placa de anclaje se ha proyectado con cartelas y redondos de acero ordinario.

El coste estimado de la pasarela de más luz: $30,912 \mathrm{~m}$ es, con beneficio industrial, de 16.310 .073 pesetas.

Todos los tipos incluyen rampas y escaleras por ambos lados con el mismo acabado que el puente. La repercusión de estos elementos es de un $46 \%$ del presupuesto total y la de la viga cajón de un $41 \%$. EL resto del coste, un $13 \%$, se distribuirá entre la cimentación y los soportes del puente. 


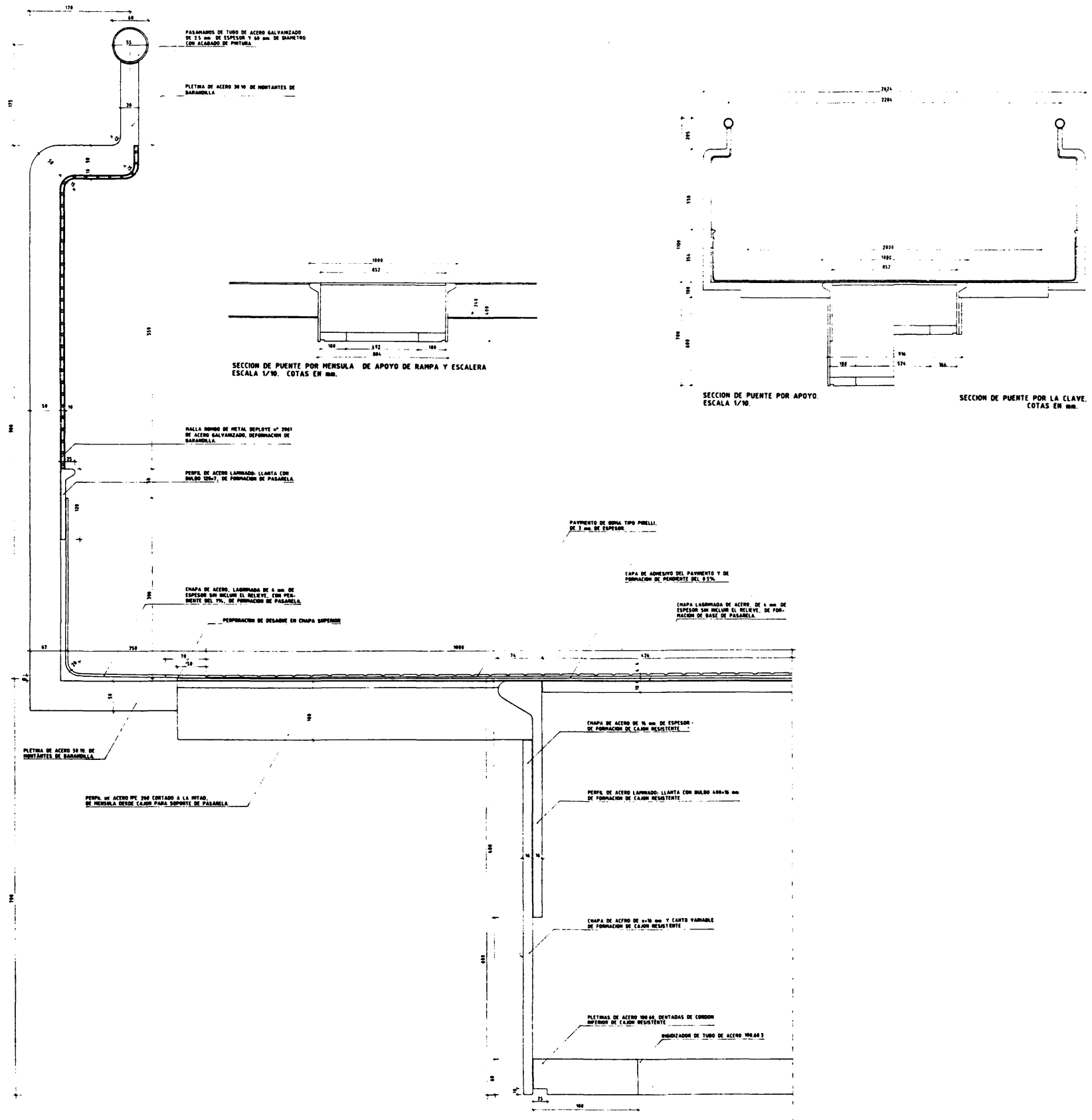

Detalle de cajón de puente y pasarela.

Se han diseñado, de igual modo unos elementos de protección sobre catenaria acordes con la estética del resto del puente.

Los sistemas de acceso a través de escaleras y rampas están igualmente descritos en los planos y están concebidos con perfiles metálicos y chapa lagrimada y barandillas del mismo tipo rematadas también con malla "deployé".

En todos los casos la unión de estos elementos, con el puente, se realiza apoyándolos sobre las ménsulas de terminación para contribuir a compensar los momentos centrales del vano principal.
En el caso de la pasarela sobre seis vías, que da una luz entre apoyos de $30,872 \mathrm{~m}$, se ha tenido que recurrir a la colocación de unos tirantes en los extremos que compensen el levantamiento de los voladizos inducido por el vano central.

El cajón resistente está constituido por chapas y perfiles de llanta tal y como indicábamos anteriormente. Las chapas son de 16 y $18 \mathrm{~mm}$ de espesor. El canto del cajón es de altura variable desde un máximo de $700 \mathrm{~mm}$ en los apoyos hasta un mínimo de $350 \mathrm{~mm}$ en la clave. Lógicamente, y dado que el sistema de luces es variable, hay tres tipos, aun cuando el aspecto exterior es homogéneo y las cantidades de material varian ligeramente en cada modelo. 


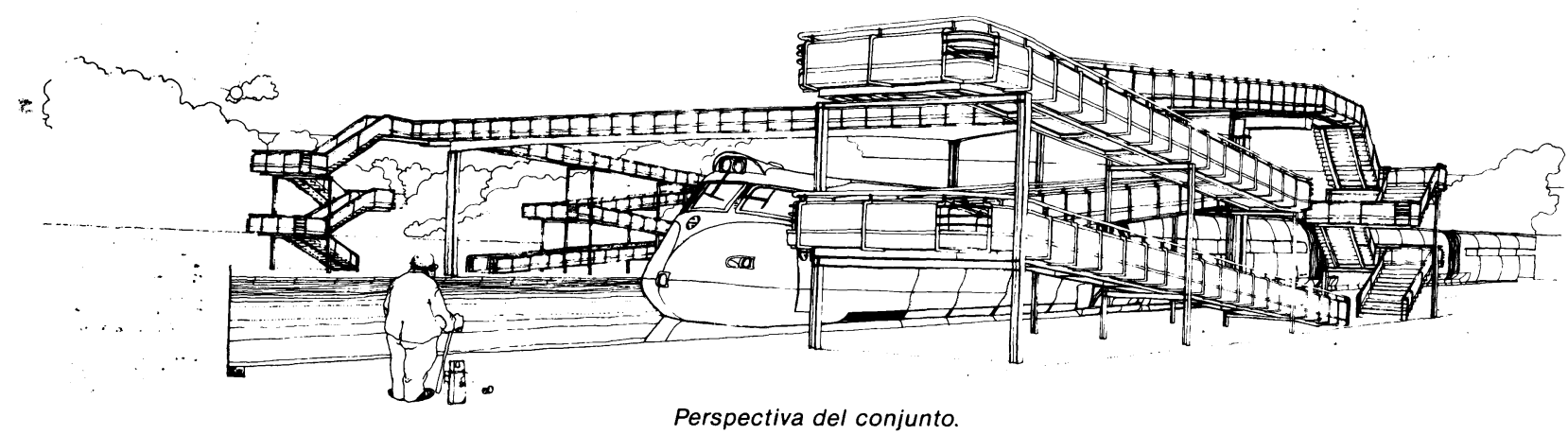

Las uniones de las piezas fundamentales, como son las pilas y el tablero de puente, se realizan con tornillería de alta resistencia.

La operación de construcción de las pasarelas cons. ta, por lo tanto, de las siguientes fases:

1. Construcción de las dos zapatas de cimentación in situ, de acuerdo con las condiciones del terreno.

2. Transporte de las pilas del puente, una vez fabricadas, y colocación de las mismas sobre los puntos de anclaje dejados para ello en las zapatas. Fijación de las piezas con redondos a la chapa, de acuerdo con lo indicado en los planos.

3. Transporte del tablero de puente que, dependiendo de la luz, se fabricará en una o dos piezas.

4. Unión, in situ, de las piezas del tablero (sólo para luces de $30 \mathrm{~m}$ ).

5. Montaje del tablero con una o dos grúas situadas fuera de las vías, en función de la luz libre de la pasarela.

6. Posterior colocación de las rampas y escaleras, fuera de las vías. Y cierre de las barandillas que no fueron montadas previamente (el montaje del tablero puede hacerse con todos los elementos de protección colocados).

7. Acabados de pintura y remates. Es preciso recordar que en la mayor parte de los casos no es nece- saria más que la presencia de una grúa. Y que todos los materiales de acabado, como son la goma del pavimento y las barandillas y tela metálica de protección, pueden llegar colocados desde la factoría.

8. Sólo en el caso de la pasarela de más luz, la de 30 $\mathrm{m}$, se necesita un andamio auxiliar de montaje para hacer que la pieza pase de un lado al otro y pue. da ser posteriormente elevada por la grúa.

Entendemos que el modelo obtenido responde a las exigencias del problema al adoptar una solución resistente y sencilla que da las prestaciones de un buen objeto industrial. Carece de peligrosidad en el uso y es de buena conservación por la facilidad en la sustitución de piezas deterioradas, problema éste que se resuelve más fácilmente en las obras metálicas como la propuesta, donde todo adquiere un sentido modular y repetitivo que hace más económico este aspecto.

Sin querer descalificar otros materiales que tienen sus ventajas, entendemos que el diseño realizado por nosotros utilizando materiales metálicos permite, con un coste similar, dar un mayor número de prestaciones y un acabado más perfecto, para proyectos como el que nos ocupa.

La facilidad de conservación permite mantener el puente con un aspecto que, en general, es difícil de conseguir con otros materiales. Como ejemplo baste recordar el buen estado en que se conservan tantas edificaciones metálicas de las estaciones y edificios del ferrocarril en general. $Y$ cómo la arquitectura del hierro ha pasado a formar parte del patrimonio histórico, en los países desarrollados.

\section{Equipo técnico}

Salvador Pérez Arroyo

Eva Hurtado Torán

Joaquin Mañoso Valderrama

Javier García Alba, Arquitectos.

Ramón Escribano Mende

Elias Llamazares de la Puente, Ingenieros. 